Aus: Proceedings of the $5^{\text {th }}$ Experimental Chaos Conference, June 28 - July 1, 1999, Orlando, Florida, Editors: M. Ding, W.L. Ditto, L.M. Pecora, M.L. Spano, World Scientific 2001

\title{
SELF-ORGANIZED QUASIPARTICLES AND OTHER PATTERNS IN PLANAR GAS- DISCHARGE SYSTEMS
}

\author{
H.-G. PURWINS, YU. A. ASTROV AND I. BRAUER \\ Institut für Angewandte Physik, University of Muenster, \\ Corrensstr. 2/4, D-48149 Muenster, Germany
}

ABSTRACT

A summary is given for the work that has been done on pattern formation in planar ac- and dc- gas-discharge systems with high ohmic and dielectric barrier respectively at the Institute of Applied Physics at the University of Muenster. WeIl defined stationary and moving solitary filaments are observed that may be referred to as selforganized quasiparticles. Among others, filaments can be scattered, generated, or annihilated, and the formation of filament clusters ("molecules") is observed. For appropriate parameters filaments in the "gaseous" phase are observed, and the condensation of large assemblies to "crystalline" phase and "liquid" phase is recorded, too. Filaments may generate superstructures e.g. domain patterns. The experimental work demonstrates that a filament is a generic pattern. In addition, reference is made to non-filamentary patterns. Finally, a list of references referring to models and numerical treatment is presented.

\section{Introduction}

The evolution of self -organized patterns in gas-discharge systems is well known since the middle of the 18th century. It is amazing to observe that so far little attention has been payed to a systematic investigation of these patterns from the point of view of modern Nonlinear Dynamics and Pattern Formation. T o fill this gap at the Institute of Applied Physics at the University of Muenster extensive experimental, theoretical and numerical investigations have been carried out for lateral acand dc-gas-dicharge systems. In this paper we mainly concentrate on the experimental results thereby emphazising spatially welllocalized large amplitude patterns that behave like quasiparticles as it turns out. A detailed discussion of the experimental results in terms of recent qualitative and quantitative models and reference to other work that is related to the work carried out at the Institute of Applied Physics will be done elsewhere.

Due to the spatial extension, because of the dissipation of electric energy and as result of the intrinsic nonlinearities of transport processes gas discharge systems may generate self-organized transient patterns and attractors. As electronic systems they can easily be driven far away from thermodynamic equilibrium. Also the formation of spatial patterns is supported by the absence of a reference system in the discharge gap in contrast e.g. to the rigid ion lattice in solid state devices. These properties make gas-discharge systems exceptional for studying self-organized patterns. 


\section{Experimental Set-Up}

The experimental investigations have been carried out with four different devices:

- A quasi -1-dimensional dc-system operated at room temperature where the edge of a thin metallic plate is opposite to the edge of a thin high-ohmic semiconductor wafer. The electrodes are separated by a discharge gap with discharge length ranging from some $100 \mu \mathrm{m}$ to some $\mathrm{mm}$. The pressure of the gas is about $10-100 \mathrm{hPa}$. The driving voltage is up to about $1 \mathrm{kV}$.

- A quasi-2-dimensional dc-system operating at room temperature with a high-ohmic semiconductor layer with diameter in the range of some $\mathrm{cm}$ parallel to a glass plate coated with ITO and being transparent with respect to the radiation emitted from the discharge gap. Roughly the discharge length is $1 \mathrm{~mm}$, the pressure $100 \mathrm{hPa}$, and the voltage up to $1 \mathrm{kV}$. $^{2}$

- A quasi-2-dimensional dc-system similar to the former one. However, to increase the resistivity of the semiconductor the latter can be cooled down to about $90 \mathrm{~K}$. In addition, the semiconductor resistivity can be controlled by an external IR-source. The discharge length ranges from about $100 \mu \mathrm{m}$ to about $1 \mathrm{~mm}$, the pressure is in the order of $100 \mathrm{hPa}$, and the voltage rises up to some $\mathrm{kV} .^{3}$

- A quasi -2-dimensional ac-system consisting of two parallel dielectric layers having a diameter in the order of several $\mathrm{cm}$ and a transparent ITO-contact at the outer sides. The dielectric plates are separated by a discharge space with a discharge length of approximately $1 \mathrm{~mm}$. The pressure is in the order of some $100 \mathrm{hPa}$ the amplitude of the driving voltage is up to some $\mathrm{kV}$, and the period is in the range of $10^{-5} \mathrm{~s}^{4}$

Among other things, self-organized patterns in the distribution of the discharge current do occur. These patterns can be observed optically due to the fact that excited states in the discharge gap emit light. Therefore, locally the current density distribution is reflected by the radiation density distribution which is approximately proportional to the current. All patterns listed below are recorded by optical means.

\section{Experimental results}

Table 1 gives a listing of patterns of the current distribution in the discharge space. These patterns consist of well-localized objects that may be referred to as quasiparticles. 


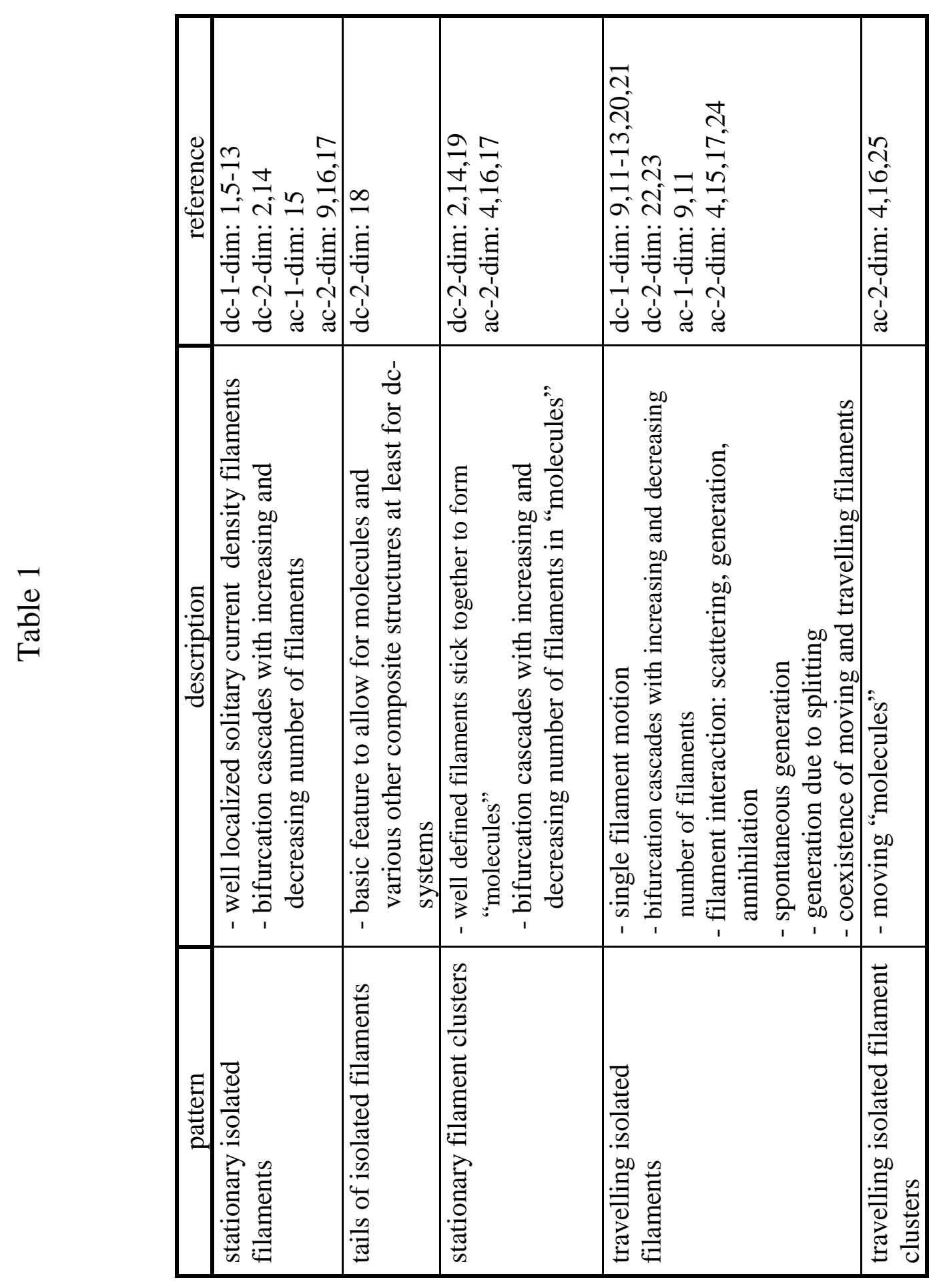




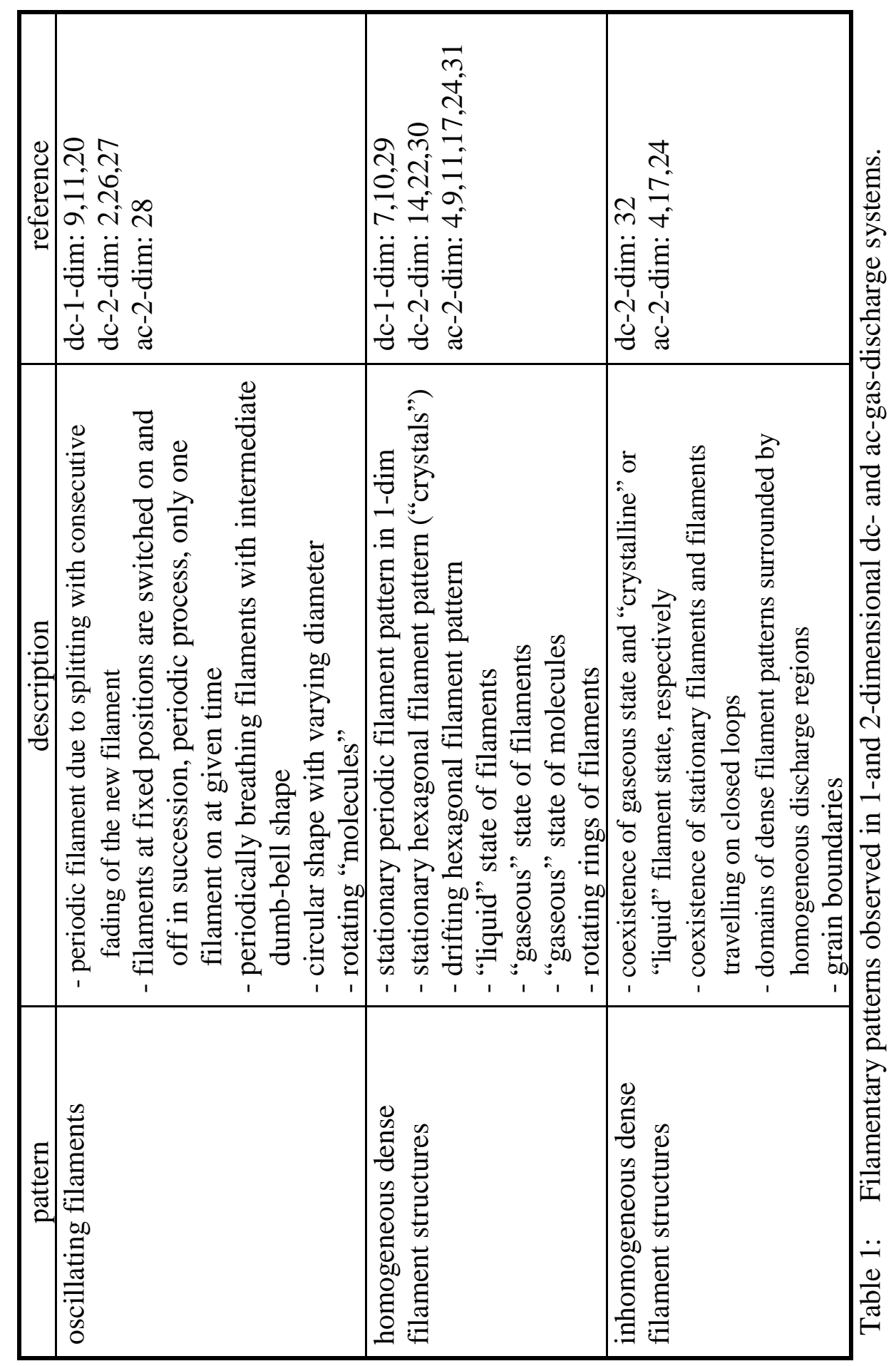




\subsection{Stationary isolated filaments}

With increasing supply voltage an increasing number of well defined isolated stationary current filaments of solitary shape may appear in a cascade of subcritical bifurcations. This is the case for ac- and dc-systems. Due to identical shape and spatial localization a "centre of mass" can be attributed to the current filaments, and they can be referred to as quasiparticles in 1- or 2-dimensional space. These filaments are the basis for a rich variety of stationary and dynamical patterns that will be discussed in what follows.

\subsection{Nonmonotonic tails of isolated filaments}

In cases where the amplitude of the current filament is high enough nonmonotonic behaviour of the filament tails can be observed experimentally. This is a generic result also obtained from model calculations and, at least in dc-systems, is considered as the basic property of single filaments to allow for higher hierarchies of structures of which the filament is the basic element.

\subsection{Stationary filament clusters}

Due to nonmonotonic behaviour of filament tails neighbouring filaments may undergo repulsive and attractive interaction depending on distance. This can lead to isolated stationary filament clusters that we refer to as "molecules". For a large number of interacting filaments the "condensed" state can be organized (see below). The stationary filament clusters are observed in dcand ac-systems.

\subsection{Travelling isolated filaments}

Isolated stationary filaments in dc- and ac-systems can undergo a bifurcation to moving filaments. Therefore, they may interact with each other, with the boundary and with inhomogeneities. The former two kinds of interactions are varified experimentally in dc- and ac-systems. Thereby, particle-like behaviour as generation, annihilation, scattering, and the formation of bound states is documented. This is the case in dc- as well as in ac-systems. In addition, the coexistence of moving and stationary filaments are observed in ac-systems. These findings strongly support the notion of a quasiparticle.

\subsection{Travelling filament clusters}

In a collision of two solitary filaments a pair of filaments can be formed. During the collision process a phase destabilization with respect to the moment of breakdown occurs.

This leads to a symmetry breaking concerning the size of the two filaments. Pairs of filaments travel at velocities of about $10 \mathrm{~m} / \mathrm{s}$ the smaller filament being the leading one. Besides pairs of filaments clusters of three and four filaments have been observed as weIl. Due to the internal symmetry of the latter clusters the velocity of these is lower than the velocity of pairs. 


\subsection{Oscillating filaments}

Besides travelling filaments, various kinds of oscillatory behaviour have been exemplified experimentally. In dc-systems, filaments may undergo splitting but the second filament is not stable and disappears. This process is repeated periodically. In dc-systems, clusters of filaments do exist in such a way that the position of single filaments is fixed, but at a given instant of time only one filament is switched on while all the others are off. In a next step the adjacent filament is on and so forth. This dynamic is repeated periodically after all filaments have been switched on once. Breathing processes of isolated filaments have been observed in ac- as well as in dc-systems. In ac-systems, one has to deal with a breathing motion between rotationally symmetrical filaments and filaments of dump-bell shape. In addition, clusters of filaments may undergo rotation in dc- and ac-systems.

\subsection{Homogeneous dense filament structures}

A variety of patterns can be considered as consisting of dense arrangements of filaments. A natural structure is a large number of filaments covering the whole fundamental active area. On a spatial scale somewhat larger than the distance between single filaments these patterns may be considered as homogeneous. They may consist of stationary or moving filaments.

Stationary arrangements of filaments forming patterns of hexagonal symmetry are observed and can be referred to as "crystals". Dense patterns of moving filaments without long range order are seen and may be called "liquids". Finally, patterns consisting of less dense, uncorrelated filaments moving irregularly all over the fundamental active area have strong similarities to "ideal gases". By changing control parameters one may induce transitions between "solid", "liquid" and "gaseous" state very similar to phase transitons in matter consisting of atoms. It is interesting to remark that the gaseous state has been realized with molecules in ac-systems, too.

In addition, hexagonal arrangements of filaments may undergo a translatory motion with constant velocity in dc- and ac-systems. Also "liquids" with constant generation of filaments at the boundary are observed and correspondingly filaments are annihilated on their way to the center of the circular fundamental area. Again this is recorded in dc- and acsystems. We also mention that dense patterns in ac-systems have been observed consisting of filaments that rotate on circles with the centre of the circle in the centre of the circular fundamental active area. The speed of rotation of rings with different radius can be different. 


\subsection{Inhomogeneous dense filament structures}

Dense filament patterns may exhibit superstructures. Recent experiments have given evidence for the coexistence of different phases e.g. of the "crystalline" and the "gaseous" state. In our findings a permanent exchange of atoms between the two aggregatstates takes place. Dense arrangements of filaments may be subdevided into stationary ones and into those travelling on closed loops that they do not leave. This is a manifestation of a memory effect. Pattens also may consist of nonstationary domains of hexagonal arrangements coexisting with regions of almost vanishing current density. "Grain boundaries" have been observed in stationary and moving hexagonal arrangements, as well.

\section{Summary}

The experimental results on the formation of filaments in planar gas discharge systems demonstrate clearly that filaments are a generic phenomenon and that the notion of a quasiparticle is a useful concept for the categorization and understanding of various stationary and dynamical patterns being self-organized in gas discharge systems. We mention that besides patterns made up of filaments, a large number of structures has been observed experimentally as there are low amplitude stripes, hexagons, target patterns and their zigzag destabilization, spiral patterns and their zigzag destabilization, strings etc. ( $1,4,13,22,33,3537)$. Also it is interesting to note that qualitative and quantitative models do exist describing many patterns and effects. E.g. isolated solitary filaments, corresponding bifurcation cascades, scattering, generation, annihilation, the formation of molecules, breathing filaments and so on are a natural outflow of these models. $(6-8,10-12,14,16,28,34,38-52)$

We mention that a lot of work has been done also with respect to structures that are not composed of filaments. With respect to this experimental work we refer to the literature. The filamentary structures have been treated analytically and numerically, as well. For this work we also refer to the literature.

\section{Acknowledgements}

We are grateful to the German BMBF and the Deutsche Forschungsgemeinschaft for supporting this work. 


\section{Rererences}

1. H. Willebrand, C. Radehaus, F.-J. Niedemostheide, R. Dohmen, and H.-G. Purwins, Observation ofSolitary Filaments and Spatially Periodic Patterns in a DC Gas Discharge System, Phys. Lett. A 149, 131 -138 (1990)

2. D. Becker, Frontausbreitung undFilamentstrukturen in einem zweidimensionalen gleichspannungsgetrieben Gasentladungssystem, Diplom-Arbeit, University of M uenster 1994

3. Y. Astrov, L. PortseI, S. Teperick, H. Willebrand, and H.-G. Purwins, Speed Properties of a Semiconductor-Discharge Gap IR Image Converter Studied with a Streak Camera System, J. Appl. Phys. 74,2159-2166 (1993)

4. E. Ammelt, D. Schweng, and H.-G. Purwins, Spatio-Temporal Pattern Formation in a Lateral High-Frequency Glow Discharge System, Phys.Lett.A 179,348-354 (1993)

5. C. Radehaus, T. Dirksmeyer, H. Willebrand, and H.-G. Purwins, Pattern Formation in Gas Discharge Systems with High Impedance Electrodes, Phys. Lett. A 125, 9294 (1987)

6. H.-G. Purwins, G. Klempt, and J. Berkemeier, Temporal and Spatial Structures of Nonlinear Dynamical Systems in: P. Grosse (Ed.), Festkörperprobleme 27,27-61 Vieweg 1987

7. H.-G. Purwins, C. Radehaus, and J. Berkerneier, Experimental Investigation of Spatial Pattern Formation in Physical Systems of Activator Inhibitor Type, Z. Naturforsch. 43a, 1729 (1988)

8. H.-G. Purwins, C. Radehaus, T. Dirksmeyer, R. Dohmen, R. Schmeling, and H. Willebrand Application ofthe Activator Inhibitor Principle to Physical Systems, Phys. Lett. A 136, 480 484 ( 1989)

9. H. Willebrand, F.-J. Niedemostheide, E. Ammelt, R. Dohmen, and H.-G. Purwins, SpatioTemporal Oscillations During Filament Splitting in Gas-Discharge Systems, Phys. Lett. A 153, 437-445 (1991)

10. F.-J. Niedemostheide, R. Dohmen, H. Willebrand, H.-J. Schulze, and H.-G. Purwins, Pattern Formation in Nonlinear Physical Systems with Characteristic Electric Properties in: Nonlinearity with Disorder, ed. F. Abdullaev, A. R. Bishop, and S. Pnevmatikos, Springer Proc. Phys. 67, 282-309 Springer 1992

11. H. Willebrand, F.-J. Niedemostheide, R. Dohmen, and H.-G. Purwins, Stationary and Dynamic Patterns ofCurrent Density in Gas -Discharge Systems in: Oscillations and Morphogenesis, ed.L. Rensing, 81-109 Marcel Dekker, 1993

12. M. Bode and H.-G. Purwins, Pattern Formation in Reaction-Diffusion Systems Dissipative Solitons in Physical Systems, Proc. Int. Conf. "Chaos, Order and Pattems: Aspects ofNonlinearity, The Gran Finale", Corno 1993, Physica D 86,5363 ( 1995)

13. E. Arnmelt, Y. Astrov, and H.-G. Purwins, Pattern Formation in Gas Discharge Systems in: SelfOrganization in Activator-Inhibitor-Systes Semiconductors, GasDischarge, and Chemical Media, ed. H. Engel, F.-J. Niedemostheide, H.-G. Purwins, and E. Schöll, Wissenschaft- und Technik- Verlag Berlin 22-27 (1996)

14. Yu. Astrov and Yu. A. Logvin, Formation of Clusters of Localized States in a Gas Discharge System via a Self-Completion Scenario, Phys.Rev.Lett 79,2983-2986 (1997)

15. T. Meierfrankenfeld, Zeitliches Verhalten eines strukturbildenden Wechselspannungsgasentladung, Diplom-Arbeit, University of Muenster 1995

16. M. Or-Guil, E. Ammelt, F.-J. Niedemostheide, and H.-G. Purwins, Pattern F ormation in Activator-Inhibitor Systems in: Pitman Research $N$ otes in Mathematics, Longman Higher Education Vol. 335,223-237, 1995

17. I. Müller, E. Ammelt, H.-G. Purwins, Interaction of Filaments in an A.C.-Driven Planar Gas Discharge System, Proc. Int. Conf. on Phenomena in Ionized Gases ICPI G XXIII T oulouse, France, 11-182 ( 1997)

18. C. Strümpel, Yu. A. Astrov, H.-G. Purwins, Observation of non-monotonic tails, results on a system with GaAs high ohmic layer at room temperature, 1999, to be published

19. Yu. A. Astrov, H.-G. Purwins, formation ofillolecules, results on a system with GaAs high ohmig layer at room temperature, 1994, to be published 
20. H. Willebrand, T. Hünteler, F.-J. Niedemostheide, R. Dohmen, and H.-G. Purwins, Periodic and Turbulent Behavior ofSolitary Structures in DistributedActive Media, Phys. Rev. A 45, 8766-8775 (1992)

21. H. Willebrand, M. Or-Guil, M. Schilke, and H.-G. Purwins, Experimental and Numerical Observation of Quasiparticle like Structures in a Distributed Dissipative System, Phys. Lett. A 177,220-224 (1993)

22. Yu. Astrov, I. Müller, E. Ammelt, and H.-G. Purwins, Zigzag Destabilyzed Spirals and Targets, Physical Review Letters 80,5341-5344 (1998)

23. Y. A. Astrov, H.-G. Purwins, Experiments following the trajectories of several filaments, simultaneously on a systeme with Si electrode at $90 \mathrm{~K}, 1999$, to be published

24. I. Brauer, E. Ammelt and H.-G. Purwins, Double Breakdowns in a Pattern Forming Dielectric Barrier Discharge System, Proc. Int. Conf. on Phenomena in Ionized Gases ICPIG XXIV Warsaw, Poland, IV-141 (1999)

25. I. Brauer, M. Bode, E. Ammelt and H.-G. Purwins, pairs of filaments of different size travel in direction of the smaller filament, measured in an ac-system, 1999, to be published

26. Y. A. Astrov, H.-G. Purwins, Breathing filaments have been observed, the measurements have been made with Si electrodes at $90 \mathrm{~K}, 1998$ to be published

27. Y.A. Astrov, H.-G. Purwins, rotating small clusters have been observed in dcsystems with Si electrode at $90 \mathrm{~K}$, siillilar to those in ac-systems, also indications of breathing mode have been observed in dc-systems again similar to observations in ac-systems, 1999, to be published

28. I. Müller, E. Ammelt and H.-G. Purwins, Self-Organized Quasiparticles: Breathing Filaments in a Gas Discharge System, Phys.Rev .Lett 82, 3428-3431 (1999)

29. C. Radehaus, H. Willebrand, R. Dohmen, F.-J. Niedemostheide, G. Bengel, and H.-G. Purwins, Spatially Periodic Patterns in a DC Gas -Discharge System, Phys. Rev. A 45,2546 2557 (1992)

30. Y.A. Astrov, H.-G. Purwins, Dense hexagonal stationary arrangements, dense nonstationary arrangements with no long-range order, less dense gaseous arrangement with no correlation and their dynamic mutual coexistence are observed. Measurements have been made with Si electrode at $90 \mathrm{~K}, 1999$, to be published

31. I. Brauer and H.-G. Purwins, rather dense molecules made of filaments may move eratically on the active area defining a "molecular gas" state, measured in an acsystem, 1999, to be published

32. Y.A. Astrov, H.-G. Purwins, filaments can form stationary hexagonal arrangements referred to as "crystals", these can coexist with the "gaseous" state, the same is observed with the "liquid" state, in both cases filaments are evaporated and condensed continuously, measurements are performed with Si electrode at $90 \mathrm{~K}, 1999$, to be published

33. H. Willebrand, K. Matthiessen, F.-J. Niedemostheide, R. Dohmen, and H.-G. Purwins, ExperimentalObservation of Simultaneous I y Existing Moving and Standing Patterns in a Gas-Discharge System, Contrib. Plasma Phys. 31,57-68 (1991)

34. G .Heidemann, M. Bode, and H.-G. Purwins, Fronts between Hopf- and Turing-Type Domains in a Two-Component Reaction-Diffusion System, Phys. Lett. A 177, 225230 (1993)

35. Y.Astrov, E. Ammelt, S. Teperick, and H.-G. Purwins, Hexagon andStripe Turing Structures in a Gas Discharge System., Phys. Lett. A 211, 184-190 (1996)

36. Y. Astrov, E. Ammelt, and H.-G. Purwins, Experimental Evidencefor Zigzag Instability of Solitary Stripes in a Gas Discharge System, Phys. Rev. Lett. 78, 31293132 (1997)

37. E. Ammelt, Y. Astrov, and H.-G. Purwins, Stripe Turing Structures in a Two Dimensional Gas Discharge System, Phys. Rev. E 55,6731-6740 (1997)

38. L.M. PortseI, Yu. A. Astrov, I. Reimann, E. Ammelt, and H.-G. Purwins, High Speed Conversion of Infrared Images with a Planar Gas Discharge System, J. Appl. Phys.85,39603965 (1999)

39. C. Radehaus, K. KardelI, H. Baumann, D. Jäger, and H.-G. Purwins, Pattern F ormation in SShaped Negative Differential Conductivity Material, Phys. BCondensed Matter 65, 515 -525 ( 1987) 
40. R. Dohmen, F.-J. Niedemostheide, H. Willebrand, and H.-G. Purwins, Analytical Approach to Stationary Wall Solutions in Bistable Reaction- Diffusion Systems, Phys.Lett. A 176,207-212 (1993)

41. M. Bode, A. Reuter, R. Schmeling, and H.-G. Purwins, Measurement of the Transition from Unito Bi- Directional Front Propagation in a Reaction-Diffusion System, Phys. Lett. A 185, 70- 76 (1994)

42. P. Schütz, M. Bode, and H.-G. Purwins, Bifurcations of Front Dynamics in a Reaction-Diffusion System with Spatial Inhomogeneities, Physica D 82,382-397

43. A. Kulka, M. Bode, and H.-G. Purwins, On the Injluence of Inhomogeneities in a Reaction-Diffusion System, Phys. Lett. A 203,33-39 (1995)

44. R. Woesler, P. Schütz, M. Bode, M. Or-Guil, and H.-G. Purwins, Oscillations of Fronts and Front $P$ airs in Two- and Three-Component Reaction- Diffusion Systems ,Physica D 91,376-405 (1996)

45. C. Schenk, M. Or-Guil, M. Bode, H.-G. Purwins, Interactingpulses in threecomponent reactiondiffusion systems on two-dimensional domains, Phys. Rev. Lett.78,3781-3784 (1997)

46. F.-J. Niedernostheide, M. Or-Guil, M. Kleinkes, and H.-G. Purwins, Dynamical behavior of spots in a nonequilibrium distributive active medium, Phys. Rev. E 55, 4107-4111 (1997)

47. C. Schenk, P. Schütz, M. Bode, and H.-G. Purwins, Interaction of Selforganized Quasiparticles in an Two-Dimensional Reaction-Diffusion System: The Formation of Molecules, Physical Rev. E 57, 6480-6486 ( 1998)

48. I. Brauer, C. Punset, H.-G. Purwins and J.P. Boeuf, Simulations of Self-Organized Filaments in a Dielectric Barrier Glow Discahrge Plasma, J. of Appl.Phys. 85, 7569- 7572 ( 1999)

49. P. Schütz, M. Bode, and v. Gafiichuk, Transitionfrom Stationary to Travelling Localized Patterns in a Two Dimensional Reaction-Diffusion System, Phys. Rev. E, 52,4465-4473 (1995)

50. M. Bode, Front propagation in inhomogeneous activator-inhibitor media, in: SelfOrganization in Activator-Inhibitor Systems: Semiconductors, Gas-Discharge and Chemical Active Media, ed. H. Engel, F.-J. Niedernostheide, H.-G. Purwins and E. Schöll, Wissenschaft \& TechnikVerlag Berlin, 200-205 (1996)

51. M. Or-Guil and M. Bode, Pattern formation in reaction-diffusion systems with relative convective flow under varying boundary conditions, in: Self-Organization in Activator-Inhibitor Systems "" Semiconductors, Gas- Discharge and Chemical Active Media, ed. H.Engel, F.-J. Niedernostheide, H.-G. Purwins and E. Schöll, Wissenschaft \& Technik Verlag Berlin, 206- 211 (1996)

52. M. Bode, Front bifurcations in reaction-diffusion systems with inhomogeneous parameter distributions, Physica D 106,270-286 (1997)

53. M. Or-Guil and M. Bode, Propagation ofTuring-Hopffronts, Physica A 249,174 178 (1998)

54. M. Or-Guil, M.Bär and M. Bode, Hierarchical patternformation, Physica A, in press 